\title{
Application of body composition zones in boys with nonalcoholic fatty liver disease
}

\author{
Minhye Choi, MD', \\ Seonhwa Lee, MD², \\ Sun Hwan Bae, MD,PhD ${ }^{1,3}$, \\ Sochung Chung, MD,PhD ${ }^{1,3}$ \\ 'Department of Pediatrics, Konkuk \\ University Medical Center, Seoul, Korea \\ ${ }^{2}$ Department of Pediatrics, College of \\ Medicine, The Catholic University of \\ Korea, Seoul, Korea \\ ${ }^{3}$ Konkuk University School of Medicine, \\ Seoul, Korea
}

Purpose: Screening nonalcoholic fatty liver disease (NAFLD) by body mass index (BMI) as a single surrogate measure for obesity has limitations. We suggest considering body composition zones by drawing a body composition chart composed of body composition indices, including BMI and percent body fat (PBF), to visualize the risk of NAFLD in obese children and adolescents.

Methods: Thirty-eight boys diagnosed with NAFLD were selected retrospectively from patients who visited Konkuk University Medical Center from 2006 to 2015. They had gone through body composition analysis by bioelectrical impedance analysis (BIA), and biochemical analyses, including a liver function test (LFT) and lipid panel, were performed. Fat-free mass index (FFMI) and fat mass index (FMI) were calculated from body composition analysis and height. We plotted FFMI and FMI of patients on a body composition chart and classified the patients into zones A to D. In addition, we analyzed the correlations between LFT, lipid panel, and body composition indices.

Results: Thirty-three of 38 boys (86.8\%) were located in zone C, corresponding to high BMI and PBF. Four boys (10.5\%) were located in zone D, which correlates with sarcopenic obesity. One boy located in zone B was a muscular adolescent. Alanine aminotransferase level was positively correlated with PBF, FMI, and BMI z-score.

Conclusion: Body composition zones on a body composition chart might be useful in risk assessment in obesity-related diseases such as NAFLD. Zones on a body composition chart could have practical applications, especially in sarcopenic obese children and adolescents.

Keywords: Nonalcoholic fatty liver disease, Childhood and adolescent obesity, Body composition, Sarcopenic obesity

\section{Introduction}

Nonalcoholic fatty liver disease (NAFLD) is the most common cause of chronic liver disease in children. ${ }^{1}$ It encompasses a spectrum of liver diseases ranging from fatty liver alone to the triad of fatty infiltration, inflammation, and fibrosis, termed nonalcoholic steatohepatitis. Early detection of NAFLD is important, and the disease can be screened using risk factors, including obesity, hypertriglyceridemia, and insulin resistance. Since it is strongly associated with obesity, NAFLD prevalence is increasing with that of obesity due to changes in lifestyle and diet patterns. ${ }^{2,3)}$ Body mass index (BMI) has been used widely as a marker of adiposity ${ }^{4)}$; however, use of BMI to identify excess body adiposity has poor sensitivity because it cannot differentiate body fat from lean mass. ${ }^{5)}$ Meanwhile, sarcopenic obesity is closely associated with chronic diseases. ${ }^{6}$ The term sarcopenia comes from the Greek words sarx (meaning flesh) and penia (meaning loss). The definition of sarcopenic obesity necessarily combines those of sarcopenia and obesity, describing those who have low muscle mass with a relatively high proportion of fat mass (FM) ${ }^{7)}$ In this paper, we suggest considering BMI and percent body fat (PBF) simultaneously to reflect body adiposity and identify at-risk patients for obesity-related

Address for correspondence:

Sochung Chung, MD,PhD

Department of Pediatrics, Konkuk University Medical Center, Konkuk University School of Medicine, 1201, Neungdong-Ro, Gwangjin-gu, Seoul 05030, Korea

Tel: +82-2-2030-7553

Fax: +82-2-2030-7748

E-mail: scchung@kuh.ac.kr https://orcid.org/0000-0002-76552691 
disease. Furthermore, we plotted the body composition indices of NAFLD patients in a chart and visualized the distribution of patient body composition. We also divided the patient group based on values of PBF and BMI and sorted them into zones to compare patient characteristics.

\section{Materials and methods}

\section{Study subjects}

Thirty-eight boys diagnosed with NAFLD were selected retrospectively from the patients who visited Konkuk University Medical Center between 2006 and 2015. The criteria of the American Association for the Study of Liver Disease were used to diagnose NAFLD. ${ }^{8)}$ Thirty-six boys presented hyperechoic liver in ultrasonography, and 2 boys showed fatty liver on computed tomography (CT). They had no factors for secondary hepatic fat accumulation such as alcohol consumption, use of steatogenic medication, or hereditary disorders. The study protocol was approved by the Institutional Review Board of Konkuk University Medical Center (Protocol No; KUH1090061). Informed consent was waived by the board due to the retrospective study design.

\section{Anthropometric measurement and body composition analysis}

Weight was measured by digital scale to the nearest $50 \mathrm{~g}$, and height was evaluated with a stadiometer to the nearest $0.1 \mathrm{~cm}$. The $z$-scores of height (HTZ), weight (WTZ), and BMI (BMIZ) were calculated based on the 2007 Korean National Growth Charts. All patients had gone through body composition analysis with bioelectrical impedance analysis (BIA) using Inbody 720 (Biospace Co., Seoul, Korea) to evaluate body fatfree mass (FFM) and FM. BMI was calculated from body weight (kg) and height $(\mathrm{m})$ using the formula BMI=weight $/(\text { height })^{2}$.

Based on the 2-compartment model, ${ }^{9)}$ body weight was calculated as the sum of FM and FFM. To evaluate the heightadjusted body indices, FMI and FFMI, these variables were substitutes for weight in the BMI equation: $\mathrm{BMI}=(\mathrm{FM}+\mathrm{FFM}) /$ height ${ }^{2}=\mathrm{FM} /$ height $^{2}+\mathrm{FFM} /$ height $^{2}=\mathrm{FMI}+\mathrm{FFMI}$. PBF was calculated by dividing FM by body weight.

\section{Body composition chart and zones}

We used the body composition chart of Hattori, ${ }^{10)}$ which is a 2-dimensional expression of FFMI and FMI, to graphically present body constitution as a quantitative measure. In addition, we plotted coordinates of FFMI and FMI, along with reference values for BMI and PBF, to visualize the distribution of patient body composition, as in previous studies. ${ }^{11-14)}$ We suggest dividing the chart into zones using values of BMI and PBF that correspond to the 85 th percentile of each subject. Values for boys at age 13 , the mean age of study subjects, are demonstrated in Fig. 1. We used the reference values of body composition indices derived from the Korean National Health and Nutrition Examination Surveys. ${ }^{15)}$ We divided the field into 4 zones: zone $\mathrm{A}, \mathrm{BMI}<23 \mathrm{~kg} / \mathrm{m}^{2}$ and $\mathrm{PBF}<30 \%$; zone $\mathrm{B}, \mathrm{BMI} \geq 23 \mathrm{~kg} / \mathrm{m}^{2}$ and $\mathrm{PBF}<30 \%$; zone $\mathrm{C}, \mathrm{BMI} \geq 23 \mathrm{~kg} / \mathrm{m}^{2}$ and $\mathrm{PBF} \geq 30 \%$; zone $\mathrm{D}$, $\mathrm{BMI}<23 \mathrm{~kg} / \mathrm{m}^{2}$ and $\mathrm{PBF} \geq 30 \%$ (Fig. 1). We also compared the biochemical profiles and body composition indices between boys in zones $\mathrm{C}$ and $\mathrm{D}$.

\section{Laboratory analysis}

Blood biochemistry analyses including liver function test (LFT) and lipid panel were performed for all patients. The LFT comprised aspartate transaminase and alanine transaminase (ALT), and the lipid panel involved total cholesterol, triglycerides, and high-density lipoprotein and low-density lipoprotein cholesterol. Patients who suffered from viral hepatitis or autoimmune hepatitis were excluded. We analyzed the correlations between blood biochemistry and body composition indices.

\section{Statistical analysis}

All analysis was performed using SPSS ver. 17.0 (SPSS Inc., Chicago, IL, USA), and a $P$-value less than 0.05 was considered statistically significant. The Mann-Whitney $U$-test was used to test for differences between zones. The relationships between biochemical profiles and body composition indices were assessed by Pearson correlation analysis.

\section{Results}

The mean age of the population of 38 boys was $13.0 \pm 2.3$-years.

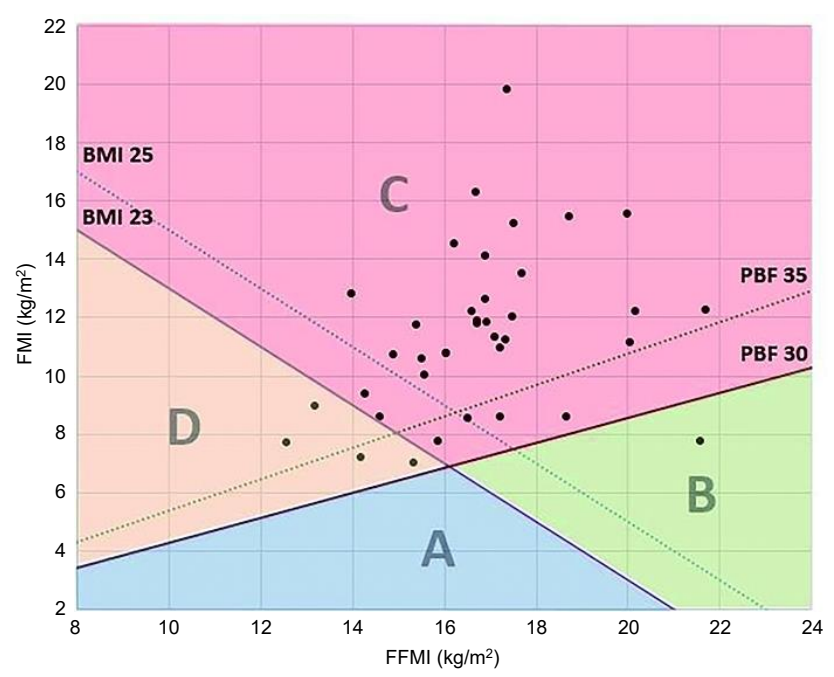

Fig. 1. The plotting of FFMI and FMI in NAFLD patients on body composition chart. The $x$-axis represents the FFMI, and the $y$-axis depicts FMI of individual. The lines of BMI and PBF were added to the same plane. NAFLD, nonalcoholic fatty liver disease; BMI, body mass index PBF, percent body fat; FMI, fat mass index; FFMI, fat free mass index. 
The mean BMI and BMIZ were 28.2 \pm 4.0 and $1.85 \pm 0.68$, respectively. Mean PBF was $39.9 \% \pm 5.5 \%$ (Table 1 ).

We expressed the body composition indices of subgroups using the body composition chart with FFMI on the $x$-axis and FMI on the $y$-axis. BMI of $23 \mathrm{~kg} / \mathrm{m} 2$ and PBF of $30 \%$, corresponding approximately to the 85 th percentile for 13 -yearold boys, ${ }^{15)}$ were chosen as reference lines on the chart, as the mean age of this study group was 13 . The plane was divided into zones A-D by the reference lines (Fig. 1). Thirty-four boys showed BMI over $23 \mathrm{~kg} / \mathrm{m}^{2}$, and 37 boys showed PBF over $30 \%$. Thirty-three boys were located in zone $\mathrm{C}$, indicating that a large percentage of NAFLD patients has both high BMI and PBF.

Table 1. The characteristics of NAFLD male patients $(n=38)$

\begin{tabular}{lc}
\hline Characteristic & Mean \pm SD \\
\hline Anthropometric data & \\
Age $(\mathrm{yr})$ & $13.0 \pm 2.3$ \\
Height $(\mathrm{cm})$ & $157.2 \pm 11.9$ \\
Weight $(\mathrm{kg})$ & $70.7 \pm 17.3$ \\
BMI $\left(\mathrm{kg} / \mathrm{m}^{2}\right)$ & $28.2 \pm 4.0$ \\
HTZ & $0.5 \pm 1.1$ \\
WTZ & $2.4 \pm 0.8$ \\
BMIZ & $1.9 \pm 0.6$ \\
Body composition & \\
FFM (kg) & $42.4 \pm 10.9$ \\
FM (kg) & $28.3 \pm 8.2$ \\
FFMl (kg/m $\left.{ }^{2}\right)$ & $16.9 \pm 2.1$ \\
FMI (kg/m $\left.{ }^{2}\right)$ & $11.4 \pm 2.8$ \\
PBF (\%) & $39.9 \pm 5.5$ \\
Biochemical profiles & \\
Alanine aminotransferase $(\mathrm{IU} / \mathrm{L})^{*}$ & $129.2 \pm 97.1$ \\
Triglyceride (mg/dL) & $153.1 \pm 101.1$ \\
HDL cholesterol (mg/dL) & $48.1 \pm 10.5$ \\
LDL cholesterol (mg/dL) & $104.9 \pm 28.8$ \\
Total cholesterol (mg/dL) & $183.6 \pm 33.6$ \\
\hline
\end{tabular}

NAFLD, nonalcoholic fatty liver disease; SD, standard deviation; BMI, body mass index; HTZ, Height z-score; WTZ, Weight z-score; BMIZ, BMI Z-score; FM, fat mass; FFM, fat free mass; PBF, percent body fat; FMI, fat mass index; FFMI, fat free mass index; HDL, high-density lipoprotein; LDL, low-density lipoprotein.

*Three patients have no acquired value.
Four boys were located in zone D, which represents sarcopenic obesity. The boy in zone B was 16 years old with a BMI of 29.3 $\mathrm{kg} / \mathrm{m} 2$, 95th-97th percentile, and PBF of $26.5 \%, 75 \mathrm{th}-85 \mathrm{th}$ percentile, probably indicating a muscular body (Table 2 ).

Furthermore, we compared the biochemical profiles and body composition indices between boys in zones $\mathrm{C}$ and D. Boys in zone $\mathrm{C}$ had higher WTZ, BMIZ, FM, FMI, FFM, and FFMI values than those of zone $\mathrm{D}(P<0.05)$. The difference in ALT between the 2 groups was not significant (Table 3 ).

In the correlation analysis between biochemical profiles and body composition indices, ALT level was positively correlated with BMIZ, PBF, and FMI. The correlation coefficients of ALT with PBF, BMIZ, and FMI were $0.498(P<0.005), 0.326$ $(P<0.005)$, and $0.433(P<0.005)$, respectively (Table 4$)$.

\section{Discussion}

NAFLD is usually asymptomatic and requires screening for detection. No screening guidelines exist outside of weight categorization (BMI $\geq 85$ th percentile for age and sex). ${ }^{16)}$ However, screening the risk population only by BMI has limitations. There is a considerable number of NAFLD patients

Table 3. Comparison of the patient characteristics of zone $\mathrm{C}$ and D

\begin{tabular}{lccc}
\hline Variable & C zone $(\mathrm{n}=33)$ & D zone $(\mathrm{n}=4)$ & $P$-value \\
\hline Age $(\mathrm{yr})$ & $13.1 \pm 2.2$ & $11.0 \pm 1.5$ & 0.056 \\
HTZ & $0.5 \pm 1.2$ & $0.7 \pm 0.5$ & 0.922 \\
WTZ & $2.5 \pm 0.7$ & $1.2 \pm 0.5$ & 0.003 \\
BMIZ & $2.1 \pm 0.5$ & $0.9 \pm 0.4$ & 0.002 \\
FM $(\mathrm{kg})$ & $30.0 \pm 7.4$ & $16.6 \pm 2.0$ & 0.002 \\
FMI $\left(\mathrm{kg} / \mathrm{m}^{2}\right)$ & $11.9 \pm 2.6$ & $7.7 \pm 0.9$ & 0.001 \\
FFM $(\mathrm{kg})$ & $43.5 \pm 10.4$ & $29.8 \pm 3.8$ & 0.007 \\
FFMI $\left(\mathrm{kg} / \mathrm{m}^{2}\right)$ & $17.1 \pm 1.7$ & $13.8 \pm 1.2$ & 0.003 \\
PBF $(\%)$ & $40.8 \pm 5.0$ & $35.8 \pm 4.1$ & 0.056 \\
ALT $(\mathrm{IU} / \mathrm{L})$ & $136.4 \pm 102.4$ & $86.8 \pm 10.1$ & 0.607 \\
\hline Va &
\end{tabular}

Values are presented as mean \pm standard deviation.

HTZ, Height z-score; WTZ, Weight z-score; BMIZ, BMI z-score; FM, fat mass; FMI, fat mass index; FFM, fat free mass; FFMI, fat free mass index; PBF, percent body fat; ALT, alanine aminotransferase.

Table 2. Clinical manifestations of patients in zone B and D on body composition chart

\begin{tabular}{|c|c|c|c|c|c|}
\hline Variable & Dzone & D zone & D zone & D zone & B zone \\
\hline Age (yr) & 13 & 10 & 10 & 10 & 16 \\
\hline BMI $\left(\mathrm{kg} / \mathrm{m}^{2}\right)$ & 21.4 & 20.3 & 22.2 & 22.3 & 29.3 \\
\hline BMIP & $50-75$ & $50-75$ & $75-85$ & $75-85$ & 97 \\
\hline PBF (\%) & 33.7 & 38 & 40.4 & 31.3 & 26.5 \\
\hline PBFP (\%) & $85-90$ & $85-90$ & $90-95$ & $50-75$ & $75-85$ \\
\hline FM (kg) & 17.8 & 16.4 & 18.3 & 13.9 & 20.4 \\
\hline FMI $\left(\mathrm{kg} / \mathrm{m}^{2}\right)$ & 7.2 & 7.7 & 9 & 7 & 7.8 \\
\hline FFM (kg) & 35 & 26.8 & 27 & 30.5 & 56.6 \\
\hline FFMI $\left(\mathrm{kg} / \mathrm{m}^{2}\right)$ & 14.2 & 12.6 & 13.2 & 15.3 & 21.6 \\
\hline ALT (IU/L) & 78 & 78 & 96 & 95 & 63 \\
\hline
\end{tabular}

BMI, body mass index; BMIP, body mass index percentile; PBF, percent body fat; PBFP, percent body fat percentile; FM, fat mass; FMI, fat mass index; FFM, fat free mass; FFMI, fat free mass index; ALT, alanine aminotransferase. 
Table 4. Correlation coefficients between ALT and body composition indices

\begin{tabular}{lcc}
\hline Variable & $r$ & $P$-value \\
\hline HTZ & -0.327 & 0.045 \\
WTZ & -0.023 & 0.892 \\
BMIZ & 0.231 & 0.046 \\
FMI & 0.433 & 0.007 \\
FFMI & -0.101 & 0.547 \\
PBF & 0.498 & 0.001 \\
\hline
\end{tabular}

ALT, alanine aminotransferase; HTZ, Height z-score; WTZ, Weight z-score; BMIZ, BMI Z-score; FMI, fat mass index; FFM, fat free mass; FFMI, fat free mass index; PBF, percent body fat. $r=$ correlation coefficient.

who maintain a weight lower than the 85th percentile for age and sex, and they usually have a large FM proportion, showing high PBF. Low muscle mass, representing sarcopenic obesity, is associated with metabolic risk, and muscular strength is positively related to higher insulin sensitivity in children and adolescents. ${ }^{17-19)}$ Early detection of sarcopenic obesity in childhood will help prevent obesity-related metabolic diseases.

In this study, although most of the NAFLD patients were located in zone C, with both high BMI and PBF, 4 NAFLD boys were located in zone $\mathrm{D}$ and might not have been identified by a screening test considering only BMI. Furthermore, one NAFLD patient was located in zone $\mathrm{B}$, showing $\mathrm{BMI} \geq 85$ th percentile but $\mathrm{PBF}<85$ th percentile, suggesting similar limitation to considering only PBF. This study implies that screening for obesity-related disease using both BMI and PBF can increase detection sensitivity. In addition, dividing body composition chart into zones could be practical at an individual person level.

Recently, new normative standards were proposed for ALT concentration ( $\leq 25 \mathrm{U} / \mathrm{L}$ for boys and $\leq 22 \mathrm{U} / \mathrm{L}$ for girls) ${ }^{20)}$ Higher ALT level suggests a more advanced stage of NAFLD, hepatitis, or fibrotic changes. ${ }^{21)}$ Thus, even though ALT elevation underestimates liver injury in NAFLD, it is still an easily available screening tool for clinicians to use when assessing children and adolescents who are overweight or obese. ${ }^{22)}$ In this study, 37 of 38 NALFD patients had ALT above the normal value ( $>25 \mathrm{U} / \mathrm{L}$ ). Furthermore, ALT showed positive correlation with BMIZ, PBF and FMI, meaning that the more obese is the patient, the more likely it is that severe liver disease is present.

BMI is an indicator of adiposity; however, both FMI and FFMI affect BMI, preventing differentiation of body fat from lean mass using BMI alone. ${ }^{5)}$ We suggest using body composition indices in evaluating obesity, which is a significant risk factor of many diseases, including NAFLD. It is important to monitor proper accretion of lean body mass including bone mineral content in children and adolescents during growth. Presenting the body composition indices using a graph and visualizing where the child is located can arouse the attention of both patient and clinician.

In this study, BIA was used for body composition analysis instead of dual-energy X-ray absorptiometry (DXA), which is often used in research and is considered the standard. BIA was used because it is a simple, easy, and time-saving technique that avoids radiation exposure and shows high correlation with other methods of obesity assessment, such as DXA and $\mathrm{CT}^{23,24)}$

There are some limitations to the study. A small group of 38 NAFLD patients was involved, and only boys were considered. A larger study considering both boys and girls should be performed in the future. In addition, waist circumference, which is a well-known risk factor of obesity-related disease, was not included among variables in this study due to the limited data.

Dividing the chart by single lines of BMI and PBF has limitations, because BMI and PBF are age- and sex-dependent, and increases in growth during the adolescent period are accompanied by increases in BMI and FFMI. ${ }^{15}$ Plotting charts for each age subgroup or age-specific assessment at the individual level should be performed to overcome this limitation.

Looking closer at the three 10-year-old boys located in zone $\mathrm{D}$, BMI of all 3 is below the 85 th percentile, but the one boy with PBF $31.3 \%$ is in the 50th-75th percentile (Table 2). When dividing the chart by reference values for a 10-year-old boy, this boy is located in zone A; however, since his PBF is greater than 30\%, he could be considered to have high PBF and needs to be carefully evaluated. In a study of children and adolescents aged 5 to 18 years, PBF above 25\% in males and 30\% in females has been reported as a significant risk factor of cardiovascular disease (CVD). ${ }^{25)}$ Overweight children and adolescents with increased CVD risk need to be evaluated according to PBF percentile subgroup even with PBF below the 85th percentile.

In conclusion, we suggest considering both BMI and PBF to reflect body adiposity and detect those at risk of obesityrelated disease such as NAFLD. Body composition zones on a body composition chart might be a useful screening tool in risk assessment, especially in sarcopenic obese children.

\section{Conflict of interest}

No potential conflict of interest relevant to this article was reported.

\section{Ethical statement}

The study protocol was approved by the Institutional Review Board of Konkuk University Medical Center (Protocol No; KUH1090061). Informed consent was waived by the board due to the retrospective study design.

\section{References}

1. Loomba R, Sirlin CB, Schwimmer JB, Lavine JE. Advances in pediatric nonalcoholic fatty liver disease. Hepatology 2009;50:1282-93.

2. Matthiessen J, Velsing Groth M, Fagt S, Biltoft-Jensen A, Stockmarr A, Andersen JS, et al. Prevalence and trends in overweight and obesity among children and adolescents in Denmark. Scand J Public Health 2008;36:153-60. 
3. Chan C. Childhood obesity and adverse health effects in Hong Kong. Obes Rev 2008;9 Suppl 1:87-90.

4. Heymsfield SB, Gallagher D, Mayer L, Beetsch J, Pietrobelli A. Scaling of human body composition to stature: new insights into body mass index. Am J Clin Nutr 2007;86:8291.

5. Okorodudu DO, Jumean MF, Montori VM, Romero-Corral A, Somers VK, Erwin PJ, et al. Diagnostic performance of body mass index to identify obesity as defined by body adiposity: a systematic review and meta-analysis. Int J Obes (Lond) 2010;34:791-9.

6. Lim HS, Park YH, Suh K, Yoo MH, Park HK, Kim HJ, et al. Association between Sarcopenia, Sarcopenic Obesity, and Chronic Disease in Korean Elderly. J Bone Metab 2018;25:187-93.

7. Stenholm S, Harris TB, Rantanen T, Visser M, Kritchevsky SB, Ferrucci L. Sarcopenic obesity: definition, cause and consequences. Curr Opin Clin Nutr Metab Care 2008;11:693-700.

8. Chalasani N, Younossi Z, Lavine JE, Diehl AM, Brunt EM, Cusi K, et al. The diagnosis and management of non-alcoholic fatty liver disease: practice Guideline by the American Association for the Study of Liver Diseases, American College of Gastroenterology, and the American Gastroenterological Association. Hepatology 2012;55:2005-23.

9. Lee SY, Gallagher D. Assessment methods in human body composition. Curr Opin Clin Nutr Metab Care 2008;11: 566-72.

10. Hattori K, Tatsumi N, Tanaka S. Assessment of body composition by using a new chart method. Am J Hum Biol 1997;9:573-8.

11. Park H, Park K, Kim MH, Kim GS, Chung S. Gender differences in relationship between fat-free mass index and fat mass index among Korean children using body composition chart. Yonsei Med J 2011;52:948-52.

12. Lee SH, Cho MH, Kim YH, Chung S. Two cases of successful type 2 diabetes control with lifestyle modification in children and adolescents. J Obes Metab Syndr 2017;26:715 .

13. Park HW, Kim YH, Cho M, Kwak BO, Kim KS, Chung $\mathrm{S}$. Adolescent build plotting on body composition chart and the type of diabetes mellitus. J Korean Med Sci 2012;27:1385-90.

14. Kim YH, Song MK, Chung S. Body composition analysis in newly diagnosed diabetic adolescent girls. J Korean Soc Pediatr Endocrinol 2011;16:172-7.
15. Park HW, Yoo HY, Kim CH, Kim H, Kwak BO, Kim KS, et al. Reference values of body composition indices: the Korean National Health and Nutrition Examination Surveys. Yonsei Med J 2015;56:95-102.

16. Huang JS, Barlow SE, Quiros-Tejeira RE, Scheimann A, Skelton J, Suskind D, et al. Childhood obesity for pediatric gastroenterologists. J Pediatr Gastroenterol Nutr 2013;56:99-109.

17. Steene-Johannessen J, Anderssen SA, Kolle E, Andersen LB. Low muscle fitness is associated with metabolic risk in youth. Med Sci Sports Exerc 2009;41:1361-7.

18. Benson AC, Torode ME, Singh MA. Muscular strength and cardiorespiratory fitness is associated with higher insulin sensitivity in children and adolescents. Int J Pediatr Obes 2006;1:222-31.

19. McCarthy HD, Samani-Radia D, Jebb SA, Prentice AM. Skeletal muscle mass reference curves for children and adolescents. Pediatr Obes 2014;9:249-59.

20. Schwimmer JB, Dunn W, Norman GJ, Pardee PE, Middleton MS, Kerkar N, et al. SAFETY study: alanine aminotransferase cutoff values are set too high for reliable detection of pediatric chronic liver disease. Gastroenterology 2010;138:1357-64, 1364.e1-2.

21. Molleston JP, Schwimmer JB, Yates KP, Murray KF, Cummings OW, Lavine JE, et al. Histological abnormalities in children with nonalcoholic fatty liver disease and normal or mildly elevated alanine aminotransferase levels. J Pediatr 2014;164:707-13.e3.

22. Styne DM, Arslanian SA, Connor EL, Farooqi IS, Murad $\mathrm{MH}$, Silverstein JH, et al. Pediatric obesity-assessment, treatment, and prevention: an endocrine society clinical practice guideline. J Clin Endocrinol Metab 2017;102:70957.

23. Lim JS, Hwang JS, Lee JA, Kim DH, Park KD, Jeong JS, et al. Cross-calibration of multi-frequency bioelectrical impedance analysis with eight-point tactile electrodes and dual-energy X-ray absorptiometry for assessment of body composition in healthy children aged 6-18 years. Pediatr Int 2009;51:263-8.

24. Yu OK, Rhee YK, Park TS, Cha YS. Comparisons of obesity assessments in over-weight elementary students using anthropometry, BIA, CT and DEXA. Nutr Res Pract 2010;4:128-35.

25. Williams DP, Going SB, Lohman TG, Harsha DW, Srinivasan SR, Webber LS, et al. Body fatness and risk for elevated blood pressure, total cholesterol, and serum lipoprotein ratios in children and adolescents. Am J Public Health 1992;82:358-63. 